\title{
An Unusual Mass at the Abdominal Site of a Ventriculoperitoneal Shunt
}

\author{
Marie-Noëlle Hébert-Blouin, Marie-Christine Guiot, Jeffery A. Hall
}

Can J Neurol Sci. 2013; 40: 263-264

The use of the peritoneal cavity for cerebrospinal fluid (CSF) diversion was introduced in 1905 by Kausch. This procedure is now a standard in the management of hydrocephalus. Welldocumented complications of the placement of the peritoneal catheter include: obstruction, infection and pseudocyst formation ${ }^{1}$. The differential diagnosis of a mass associated with an abdominal surgical scar includes: fibrosis (scar or keloid), hernia, suture granuloma, abscess and in women of reproductive age, endometrioma ${ }^{2}$. Here we report on a young woman with a mass at the abdominal site of her ventriculoperitoneal shunt that was surgically excised and histologically proven to be an endometrioma.

\section{Case Report}

A 21-year-old woman with a history of meningocele and hydrocephalus treated perinatally, required a shunt revision two years prior to presentation for a catheter break at the level of her neck. At that time, both the scalp and abdominal incisions had been reopened. Approximately one year after the revision, she began to notice a lump at the site of her abdominal incision. She reported the mass to fluctuate in size and tenderness every few weeks.

Neurological examination was unchanged from baseline. The valve filled and emptied well. There was no tenderness along the shunt tract. The abdomen was benign. A firm, slightly-tender mass, in close proximity to the superior and lateral aspect of the abdominal incision in the right upper quadrant was easily palpated.

A computed tomogram (CT) of the head showed a right transparietal ventricular catheter well positioned in the lateral ventricle. A radiographic shunt series demonstrated continuity of the system. An abdominal ultrasound showed a 1.6 x $1.8 \mathrm{~cm}$ fluid collection adjacent to the tube beneath the skin but superficial to the abdominal wall with no obvious extension into the peritoneal cavity.

We elected to perform an exploration and/or excision biopsy of the lesion. At the time of surgery, a hard, non-fluctuant mass was encountered lateral to the insertion site of the peritoneal catheter. Several chocolate-brown cystic nodules were entered with brownish fluid extrusion. Stat gram stain revealed: 3+ WBC, 4+ RBC, 2+ epithelial cells and no organisms. The lesion was then carefully surrounded and dissected en bloc. Pathology yielded endometrioma.

Post-operatively she was assessed by the gynecology service who found no evidence of pelvic or extra-pelvic endometriosis by CT imaging. She was advised to take an oral contraceptive pill, since it is often used to treat young women with mild endometriosis who also require effective contraception. She has done so for two years; more than six years have passed since she stopped the contraceptive pill and she has no obvious recurrence.

\section{Surgical Pathology}

Histopathology revealed a fibrous stroma containing some irregular shaped glandular formations surrounded by an edematous and cellular stroma. The glands were lined with a columnar epithelium which appeared focally pseudostriated. The cells had characteristic elongated nuclei with dense chromatin (Figure 1A). The epithelium stained strongly for cytokeratin (Figure 1B). The nuclei were positive for estrogen (Figure 1C), and progesterone (Figure 1D) receptors. In adjacent tissues, collections of macrophages filled with hemosiderin were seen. The histological diagnosis of endometrioma requires two of the following three features: endometrial-like glands, endometrial stroma and/or hemosiderin pigment ${ }^{3}$. In this case, all features were present.

\section{DiscuSSION}

The literature describes the spectrum of complications associated with ventriculo-peritoneal shunting, including infection, obstruction, pseudocyst formation, abdominal wall, bowel and bladder perforation, volvulus, transdiaphragmatic migration, scrotal extrusion, umbilical fistula and an increased incidence of inguinal hernia ${ }^{1}$. There is a report of a shunted patient who developed a painful umbilical mass but its relationship to the surgical scar is unclear and could represent previously described endometrioma associated with umbilical hernia $^{2}$. To our knowledge, the presence of an endometrioma at the site of the abdominal incision for the peritoneal catheter, distant from the umbilicus, has not been reported.

Endometriosis, defined as uterine mucosa outside of the uterine cavity, affects $10 \%$ of menstruating females ${ }^{4}$. It has been described in almost all body cavities and organs. Endometrioma, defined as extra-pelvic endometriosis forming a discrete mass, is rare but appears to have a predilection for abdominal scars. The most common site is in association with Cesarean section with

From the Departments of Neurosurgery (MNHB, JAH), Pathology (MCG), Montreal Neurologic Hospital and Institute, McGill University, Montreal, Quebec, Canada. Received February 14, 2011. Final Revisions Submitted SePtember 11, 2012. Correspondence to: Jeffery A. Hall, Montreal Neurological Hospital, Department of Neurosurgery, McGill University, 3801 University St, Suite 122C, Montreal, Quebec, H3A 2B4, Canada. Email: jeff.hall@mcgill.ca. 


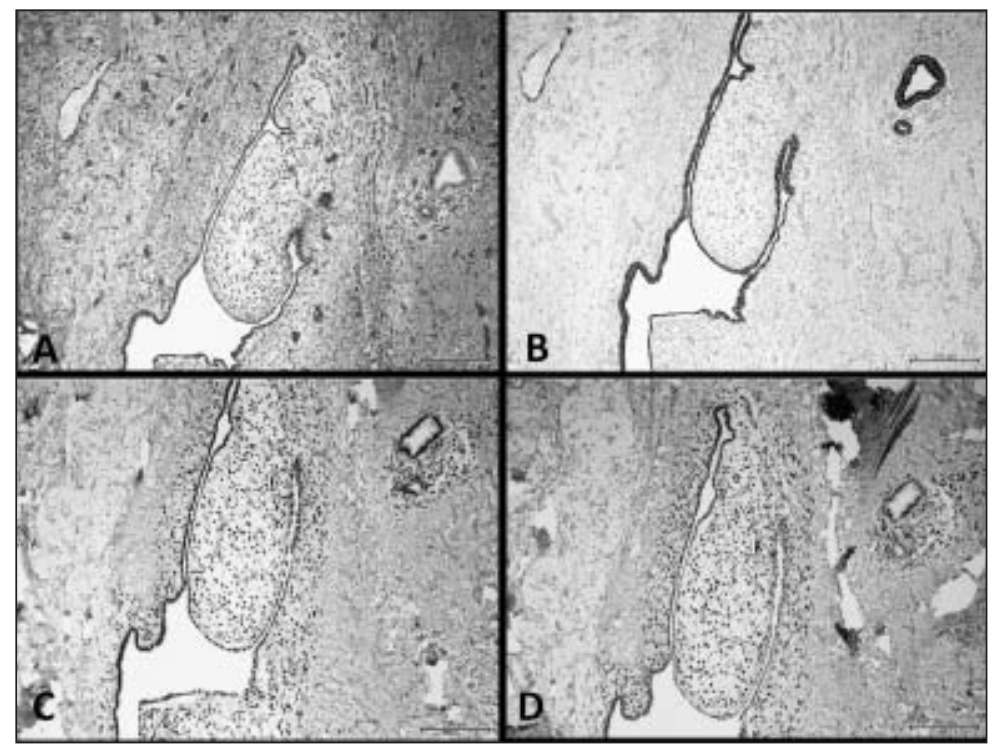

Figure: A. Irregular gland formations embedded in dense fibrous stroma. Hematoxylin and eosin $(H \& E)$ stain, $x 100 . B$. The epithelium of the glands is strongly positive for Cytokeratin AE1/AE3 (Ventana), x100. C. The nuclei are strongly positive for estrogen receptors (Ventana, clone SP1), x100. D. The nuclei are positive for progesterone receptors (Ventana, clone 1E7), x100.

an incidence of 0.2 to $1 \%$. Endometrioma has also been reported in the needle tract of amniocentesis and episiotomy. This may reflect spillage of uterine mucosal cells at the time of uterectomy, uterotomy or delivery. It does not, however, help explain endometrioma reported in cases associated with appendectomy scars, inguinal herniorraphy, laparoscopic trocar tracts, or the case presented herein, in which no apparent penetration of the uterus occurred.

The majority of patients with endometrioma present with a painful mass at the site of a gynecologic procedure. The pain and size of the mass may fluctuate with the patient's menstrual cycle $^{2}$, as was the case with our patient. Interestingly, it is the minority $(25.9 \%)$ of patients with endometrioma who have concomitant pelvic endometriosis ${ }^{5}$. The median age reported in the literature at the time of diagnosis is 28.4 to 34 years $^{2,3}$. The published interval from surgery to onset of symptoms is between 6 months to 20 years $^{2,3}$. Where reported, the preoperative diagnosis has been missed in 40 to $80 \%$ of patients ${ }^{2}$.

Multiple diagnostic procedures are employed to diagnose endometriosis and endometrioma. Ultrasound will detect solid or cystic components and may be used to track growth or regression over time. A CT and magnetic resonance imaging (MRI) may be helpful to demonstrate the extent of the disease and fine needle aspiration may aid the diagnosis. Many consider wide excision with a margin of at least one $\mathrm{cm}$ to be the treatment of choice, even in recurrent disease ${ }^{2,5}$. Hormonal treatment may be associated with androgenic side-effects and symptoms would be expected to recur with cessation of treatment. In our patient, the benefit and required duration of a hormonal therapy after total resection of the lesion and no evidence of endometriosis on CT is unclear. Despite cessation of her hormonal therapy, she had no obvious recurrence.

The etiology of endometriosis and endometrioma is not yet fully understood. Plausible hypotheses are 1) cell transport in which uterine mucosal tissue is thought to migrate from the uterus, 2) metaplasia of the pelvic peritoneum which supports that primitive pluripotent cells undergo specialized differentiation to become uterine tissue in situ, and 3) shed endometrium may release tissue factors that can induce undifferentiated mesenchyme to form endometrial tissue. Either theory alone or some hybrid could be used to explain the endometrioma in our patient. Uterine mucosal cells in the peritoneum could have migrated along the distal catheter; however, our patient had no evidence of pelvic endometriosis, as is the case for the majority of patients presenting with endometrioma. It is also possible that our patient had pelvic endometriosis not detected by the imaging technique used, i.e. $\mathrm{CT}$, which is fairly insensitive and may not be the imaging of choice for the diagnosis of pelvic endometriosis. Another etiology could explain the endometrioma in our patient: precursor cells in or around the site of insertion of the peritoneal catheter may have undergone metaplastic changes. It is tempting to conclude that cells, be they primitive and pluripotent or fully differentiated, may be activated in such a way by the local biochemical environment of a healing wound that predisposes or permits continued growth and eventual formation of endometrioma.

\section{REFERENCES}

1. Bryant MS, Bremer AM, Tepas JJ 3rd, et al. Abdominal complications of ventriculo-peritoneal shunts. Case reports and review of the literature. Am Surg. 1988;54(1):50-5.

2. Patterson GK, Winburn GB. Abdominal wall endometriomas: report of eight cases. Am Surg. 1999;65(1):36-9.

3. Dwivedi AJ, Agrawal SN, Silva YJ. Abdominal wall endometriomas. Dig Dis Sci. 2002;47(2):456-61.

4. Guidice LC. Genomics' role in understanding the pathogenesis of endometriosis. Sem Repro Med. 2003;21(2):119-24.

5. Rani, PR, Soundararaghavan S, Rajaram, P. Endometriosis in abdominal scars: review of 27 cases. Int J Gynecol Obstet. 1991; 36(3):215-8. 